\title{
Amenaza y persuasión en mensajes de salud sobre consumo de alcohol: de la teoría a la práctica*
}

\author{
Ordoñana Martín, J.R.; Gómez Amor, J. \\ Área de Psicobiología. Dep. Anatomía Humana y Psicobiología. Área de Psicobiología. Universidad de Murcia
}

Juan Ramón Ordoñana. Departamento de Anatomía Humana y Psicobiología. Área de Psicobiología. Facultad de Psicología. Universidad de Murcia. 30100 Murcia. ordonana@um.es

\section{RESUMEN}

La información sobre el alcohol y sus consecuencias proviene de dos polos contrapuestos. De un lado los mensajes acerca de los beneficios de beber alcohol provenientes de la publicidad, grupo de amigos o el ambiente social en general. De otro, la información proporcionada por los profesionales e instituciones sanitarias, basada, en su mayor parte, en exponer posibles consecuencias negativas del consumo con la intención de reducirlo o controlarlo. La utilización de la amenaza en un grado u otro es, por tanto, un recurso habitual en los mensajes acerca de los riesgos de consumir alcohol dirigidos a los jóvenes.

La presencia de información amenazante, sobre riesgos, en los mensajes preventivos y de promoción de salud, resulta difícilmente evitable y puede tener tanto efectos positivos como negativos dependiendo de sus características. En consecuencia el planteamiento actual se dirige no tanto a evitar la amenaza, puesto que es difícilmente eludible, sino a determinar cuáles son las condiciones bajo las que la amenaza ejerce un efecto positivo sobre la adherencia a las recomendaciones.

Para que la amenaza tenga mayor probabilidad de producir un efecto positivo deberían cumplirse algunas condiciones: debe servir para generar una respuesta atencional, que se apoye en argumentos relevantes y que vaya acompañada de una recomendación percibida como eficaz para eliminar el riesgo. Por otra parte, la eficacia de los mensajes dependerá también de otros factores como la utilización de distintos estilos, la variación en los temas y argumentos para mantener la atención, la utilización de información verídica, relevante y verosímil para la audiencia o la atención a las características específicas de la población a la que se dirigen.

Por último, se hace hincapié en que disponemos de información suficiente sobre los comportamientos, creencias y actitudes de los jóvenes acerca del alcohol, a lo que se añaden orientaciones válidas para generar mensajes más eficaces, siempre que tengamos una idea clara de lo que queremos transmitirles.

Palabras clave: mensajes de salud, mensajes de miedo, riesgo, comunicación, jóvenes, campañas, prevención.

\section{ABSTRACT}

Information about alcohol and its consequences comes from two opposing poles. On the one hand, messages about the benefits of drinking alcohol coming from publicity, group of friends or the social milieu in general. On the other hand, the information provided by professionals and health authorities, which is based, mainly, in explaining the possible negative consequences of consumption, with the intention of reducing or controlling it. Therefore, use of threat, in different degrees, is a common resource in the communications about the risks of drinking alcohol designed for young people.

Presence of threatening information, in health promotion and prevention messages, is hardly avoidable and can lead to both positive and negative results, depending on its characteristics. In consequence current models are not dealing with the dichotomy threat-not threat, but instead, are trying to determine the conditions under which threat exerts a positive effect on the adherence to message recommendations.

In order for threat to increase the probability of producing a positive effect some basic conditions should be met: it needs to generate an attentional response; it must lean on in strong arguments and has to be accompanied by a recommendation perceived as effective to eliminate the risk. On the other hand, message effectiveness will also depend on other factors like use of different message styles, variation in topics and arguments to maintain attention, use of truthful and relevant information or taking into account the specific characteristics from the target group.

Finally it is stressed that we have enough information about young people's behavior, beliefs and attitudes about alcohol, and that we have useful orientations to generate more effective messages, whenever we have a clear idea of what we want to transmit to them.

Key words: health messages, fear-appeals, risk, communication, adolescents, campaigns, prevention.

\footnotetext{
* La realización de este trabajo ha sido financiada por la Delegación del Gobierno para el Plan Nacional sobre Drogas (Convocatoria para la concesión de ayudas económicas para el desarrollo de proyectos de investigación sobre drogodependencias. Orden de 18 de mayo de 2001)
} 


\section{INTRODUCCIÓN}

$\mathbf{L}$ a situación que se encuentra el profesional sociosanitario a la hora de llevar a cabo una intervención educativa, o meramente informativa, sobre el alcohol en los jóvenes está definida por dos extremos contrapuestos.

De un lado la omnipresente publicidad cuyo efecto, aunque discutido y difícil de probar, continúa ligando el consumo de bebidas alcohólicas a la desaparición de emociones negativas, la inducción de estados de bienestar (despreocupación, placer,...) y el éxito social y sexual ${ }^{1,2}$. A ésta se añaden la poderosa influencia del grupo de amigos, la asociación del consumo de alcohol a diversión y comunicación, y una red de intereses económicos de pequeños empresarios que, conjuntamente, forman el escenario en que se desenvuelve la vida social de la mayoría de la juventud en nuestra sociedad $^{3}$.

Del otro lado, con intervenciones bienintencionadas pero, en su mayor parte, esporádicas, de corta duración y con escasez de recursos, algunos mensajes de profesionales e instituciones sanitarias y educativas. Mensajes que intentan ofrecer una perspectiva diferente; tratando de proporcionar información "ágil, clara y veraz de los diferentes aspectos relacionados con el consumo de drogas" ${ }^{4}$ y con el ánimo de ofrecer conocimientos y recursos que capaciten al joven para que escoja la alternativa más saludable.

El objetivo de toda esta intrincada red de informaciones y mensajes es básicamente el mismo: tomar preponderancia sobre el resto y hacerse presente en el momento en que el individuo va a tomar una decisión ¿Bebo o no bebo? ¿Qué tomo? ¿He bebido ya suficiente/demasiado? ¿Cómo me encuentro? ¿Qué pasa si no bebo? ¿Gano algo? ¿Qué pierdo? De la evaluación de todas estas cuestiones, realizada de forma más o menos consciente, surgirá un comportamiento concreto y este mismo proceso se repetirá en cada ocasión en que el joven tenga que tomar una decisión.

Influir* en esta toma de decisiones de una persona es, por tanto, un proceso complejo y multifactorial en el que, a la vista de los recursos invertidos y su constante presencia, los mensajes acerca de las bondades del consumo tienen, a priori, las de ganar. $Y$, sin embargo, los mensajes sobre alcohol y salud, de alguna forma, llegan a los jóvenes. Las intervenciones breves, ya sean en el marco de la asistencia sanitaria o en el más abierto de centros educativos, se han mostrado eficaces y han conseguido reducciones significativas, incluso a largo plazo, en la frecuencia de las conductas relacionadas con el consumo de alcohol y riesgos asociados $5,6,7,8,9$. Esta evidencia nos lleva a dos conclusiones iniciales: Primero, que a pesar de la importante influencia de factores favorecedores del consumo de alcohol, la información sobre salud encuentra vías para estar presente en alguna de las decisiones del individuo; y segundo, que determinar qué factores facilitan que este tipo de información surja y esté presente en el momento de la toma de decisiones es crucial para potenciar el efecto de las intervenciones. Efecto que, por otra parte y siendo realistas, siempre será de un alcance limitado y dependerá de los recursos invertidos y de su interacción con otros elementos de índole social.

¿Cuáles son entonces los factores que influyen en la mayor o menor probabilidad de éxito de una comunicación, tanto a través de medios de comunicación de masas como en interacciones cara a cara? ¿Cómo debe el profesional o las instituciones sanitarias transmitir los mensajes, de forma que cumplan los requisitos de ser éticos, relevantes y eficaces? Las respuestas a estas preguntas tienen, obviamente, una aplicación práctica inmediata en la promoción de salud y la prevención del consumo de drogas, en general, y del alcohol en particular. Una de las principales actividades de los programas y servicios de prevención consiste en la planificación y puesta en marcha de estrategias de comunicación destinadas a transmitir información, promover actitudes y capacitar a los destinatarios para tomar decisiones responsables y saludables en torno al consumo.

Esta cuestión, de hecho, aparece reflejada en el propio documento de la "Estrategia Nacional sobre Drogas 2000-2008", donde el Área de Comunicación Social representa un ámbito de intervención por sí misma pero, además, es considerada fundamental por su transversalidad al resto de las intervenciones ${ }^{4}$. Es evidente, por tanto, que la optimización de los mensajes de salud que se transmiten es un importante elemento, aunque por supuesto no el único, para la consecución de los objetivos de los programas de salud pública.

\section{INFORMACIÓNY RIESGO}

En todo caso, a pesar de su importancia, la solución a estas cuestiones no es fácil y ha sido objeto de un creciente interés científico por profundizar en los mecanismos a través de los cuáles se recibe, procesa y analiza la información recibida por los jóvenes.

\footnotetext{
* El objetivo de este texto no es profundizar en los aspectos éticos de esta cuestión. Influir no debe entenderse aquí como sinónimo de manipular. Asumimos que el objetivo de todo mensaje de salud es educar y capacitar a la persona esperando que, con toda la información disponible y desde su libertad individual, escoja la alternativa conductual más ventajosa desde el punto de vista de su salud.
} 
La literatura sobre mensajes persuasivos ha trabajado sobre diferentes elementos que pueden afectar a la capacidad de una comunicación de mostrar un impacto en la audiencia. Estos elementos incluyen aspectos relacionados con la fuente de la información, el tipo de mensaje utilizado (forma de presentación y argumento), el tipo de conducta objeto de la intervención. las características de la audiencia, factores situacionales o la experiencia previa de exposición a mensajes ${ }^{10,11 .}$

Y entre estos elementos, el concepto de riesgo o amenaza para la salud, y la forma de presentación de dicha amenaza, tienen un papel central por dos cuestiones fundamentales: su ambivalencia potencial y su inevitabilidad.

Ambivalencia potencial: Algunos autores han apuntado que la utilización de la amenaza como argumento puede conllevar el rechazo del mensaje, por lo que sería más apropiado utilizar mensajes de tipo positivo ${ }^{12,}$ ${ }^{13}$. No obstante, sin cuestionar la posible necesidad de mensajes de estas características, las últimas revisiones coinciden en la probada eficacia de utilizar la amenaza para incrementar la adherencia al mensaje, aunque bajo determinadas condiciones ${ }^{10,14,15,16,17,18,19}$.

Es decir, la utilización de la amenaza sería apropiada, pero no en cualquier ocasión, ante cualquier audiencia o con cualquier intensidad; y dependiendo de las circunstancias en que se presente podría dar lugar a resultados tanto positivos como negativos, o incluso pasar desapercibida.

Inevitabilidad: El riesgo siempre está presente en las comunicaciones sobre salud. De manera explícita o implícita se habla del riesgo que determinadas acciones o conductas tienen para la salud del individuo o la población. La presencia de una amenaza, más o menos velada, es difícilmente evitable y, aunque con grandes variaciones en cuanto a su magnitud, su gravedad, su orientación o el nivel de agresividad de la forma en que se presentan, el usuario se ve confrontado habitualmente con una serie de riesgos que debe aceptar o evitar en función de las circunstancias.

Esta característica hace que esta cuestión se halle presente en cualquier forma de comunicación entre los profesionales sanitarios y la población. Ya sea en los medios de comunicación, a través de materiales educativos impresos, o en las interacciones persona a persona que se producen en las consultas individuales, los riesgos para la salud, sus consecuencias y la forma de evitarlos o afrontarlos constituyen el eje central del proceso de Educación para la Salud ${ }^{20,21}$.

\section{AMENAZA Y PERSUASIÓN}

En consecuencia con lo anterior, el planteamiento actual no es tanto, amenaza o no amenaza, sino cuán- do, cómo y a quién presentar esa amenaza. Es decir, la pretensión se dirige no tanto a evitar la información amenazante, dado que es difícilmente eludible cuando se habla de efectos y consecuencias, sino a determinar cuáles son las condiciones bajo las que la amenaza ejerce un efecto positivo sobre la adherencia a las recomendaciones ${ }^{12,16}$.

En la búsqueda de esas condiciones, hay que tener en cuenta que la amenaza y la forma de presentarla juegan un doble papel: inciden en el efecto inicial que la comunicación produce en el sujeto y afectan a la relevancia del argumento utilizado. En otras palabras, supuestamente, cuando una persona es expuesta a un mensaje éste debe, en primer lugar, provocar una reacción en el sujeto. A continuación, y dependiendo de esa primera respuesta, se procesará la información contenida en la comunicación. Así, el papel inicial de una presentación con imágenes impactantes o una mención explícita a consecuencias indeseables sería el de generar una respuesta en el sujeto que motive la retención y la reflexión posterior sobre los contenidos del mensaje.

Este planteamiento se recoge, en sus postulados básicos, en el Modelo de Respuesta Paralela Extendido de Witte ${ }^{14,15,22}$. De acuerdo con este intento de explicación teórica, cuando una persona se enfrenta a un mensaje de miedo se inician dos evaluaciones. Primero, el individuo evalúa la amenaza percibida, la cual dependería de la severidad de la amenaza y de la susceptibilidad a la misma que percibe el sujeto. Es decir, "¿Hasta qué punto la consecuencia es negativa?" y "¿Qué probabilidad hay de que me afecte a mí directa o indirectamente?" Ambos factores estarían afectados, evidentemente, por la forma como se haya presentado la información en la comunicación. En este punto, si la amenaza es percibida como trivial o irrelevante no habría motivación para seguir procesando el mensaje, la eficacia de la recomendación no sería evaluada y no se produciría respuesta al mensaje de miedo.

Si, por el contrario, esta primera evaluación concluye con una percepción de amenaza moderada o fuerte se motivaría la segunda evaluación, que se centraría en la eficacia de la acción recomendada en el mensaje, la cual estaría compuesta a su vez de su eficacia para evitar la amenaza y la auto-eficacia o confianza en la propia capacidad de llevarlas a cabo: "¿Evito el riesgo si sigo las recomendaciones?" "¿Puedo y me resulta fácil seguir estas recomendaciones?"

En esta segunda evaluación, puede suceder que la eficacia percibida de la acción recomendada sea baja, en cuyo caso se desencadenarían respuestas defensivas de rechazo o minimización. Sin embargo, si se percibe que esta eficacia es alta, se facilitarían las respuestas de protección. 
Partiendo de este Modelo, e incorporando aportaciones de otras formulaciones actuales, podemos decir que la respuesta inicial ante un mensaje de esta clase puede ser de dos tipos diferentes según las características de la audiencia, del entorno y del problema en sí: puede generar una respuesta atencional, provocando interés en el contenido, o defensiva/emocional, como miedo, ira o asco ${ }^{10,12,23}$. Estas dos formas de reacción dan lugar a respuestas psicofisiológicas diferenciadas que pueden ser observadas en laboratorio. En este sentido, trabajos recientes de nuestro grupo de investigación han encontrado diferentes patrones de activación psicofisiológica, sugiriendo distintos tipos de respuesta, ante mensajes con distintos

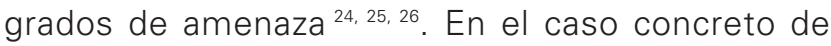
mensajes de alcohol dirigidos a jóvenes, los resultados apuntan hacia una mayor frecuencia de aparición de respuestas de tipo atencional ante un mensaje de amenaza, comparado con otros tipos de estímulo. Por otra parte, el mensaje de amenaza produjo una mayor percepción de riesgo y de la eficacia de no consumir alcohol para mejorar/preservar la salud en los sujetos. Sin embargo, no encontramos diferencias entre mensajes en cuanto a valoración del mismo (claridad, objetividad, ...) o percepción de manipulación ${ }^{27}$.

Esta diferenciación entre respuestas atencionales y defensivas podría explicar la ambivalencia que presenta la utilización de la amenaza de la que hablábamos antes. Si ésta genera principalmente una respuesta atencional se lograría el objetivo de centrar los recursos del sujeto sobre la información, favoreciendo así la retención y la reflexión sobre la misma. Si lo que genera es una respuesta defensiva de tipo emocional, se corre el riesgo de que se preste atención a la emoción en sí y no al contenido del mensaje.

La probabilidad de producir una u otra variará según los factores ya mencionados pero hay que recordar que en una cultura de la imagen como la nuestra, en la que los mensajes impactantes en imágenes y sonido están presentes continuamente en los medios de comunicación de masas, se requiere un tono elevado para producir respuestas emocionales, sobre todo, en los jóvenes. La habituación no les hace completamente insensibles a estos impactos, pero disminuye la respuesta y, de hecho, la información destinada a producir miedo en la audiencia, parece producir en realidad atención en buen número de sujetos.

Posteriormente, una vez conseguida la atención, el foco pasaría al procesamiento de la información que contiene el mensaje. Es decir a la relevancia del argumento utilizado y la utilidad de las recomendaciones que reforzarían o minimizarían el efecto inicial.

\section{DE LA TEORÍA A LA PRÁCTICA}

En el caso concreto del alcohol, la utilización de información sobre riesgos ha sido y es moneda corrien- te. Son comunes los mensajes que utilizan la amenaza con consecuencias indeseables, si no se siguen las recomendaciones propuestas, para inducir a una conducta determinada. Esta amenaza puede presentarse de maneras diferentes: como el resultado directo de una conducta o como la pérdida de consecuencias positivas; puede ser abierta o sutil; y puede centrarse en diferentes aspectos que afectan al individuo como salud, auto-estima o relaciones interpersonales.

Por este motivo, a pesar de que no tenemos aún respuesta para todas las preguntas formuladas, resulta de especial interés aplicar los conocimientos disponibles sobre los factores que hacen más eficaz un mensaje de estas características. Conocimientos, que no solo son aplicables a este caso, sino a cualquier problema de salud en el que la transmisión de información a la población tenga un papel fundamental.

De esta forma, se resumen a continuación algunas de las cuestiones que, a la luz de los conocimientos actuales, se deberían tener en cuenta en el momento de utilizar información de este tipo.

\section{¿Es eficaz utilizar mensajes de amenaza?}

-En primer lugar, utilizar información amenazante es difícil de evitar, sobre todo en comunicaciones largas y que hacen referencia a las consecuencias para la salud de una conducta, como en el caso del consumo de alcohol. Dada esta circunstancia, lo importante es tratar de utilizarla adecuadamente.

Por otra parte, esto no quiere decir que este sea el único tipo de mensaje posible. La utilización de otros modelos, por ejemplo, asociando el no consumo a sentimientos positivos, no es incompatible y, de hecho, la ampliación del espectro de temas y estilos incrementará, probablemente, las posibilidades de llegar a la audiencia ${ }^{28}$.

- La percepción de un cierto riesgo parece ser un factor importante en el desarrollo posterior de conductas preventivas. El conocimiento de la existencia de una amenaza tiene un papel particularmente relevante cuando lo que se pretende es un cambio actitudinal o conductual. En estos casos, la certeza de la existencia de un riesgo para la salud resulta necesaria, aunque no suficiente, para promover la adopción de conductas saludables y adaptadas ${ }^{29}$.

-Ahora bien, la utilidad de la información amenazante en los mensajes de salud está limitada por una serie de factores. Es decir, tendría un efecto positivo sólo si se cumplen algunas condiciones básicas: que genere una respuesta atencional, que se apoye en argumentos relevantes y que vaya acompañada de una recomendación percibida como eficaz para eliminar la amenaza. 


\section{¿Qué respuesta deben producir en la audiencia?}

-Como hemos dicho, el mensaje debería producir en un primer momento una respuesta atencional en lugar de una respuesta defensiva de tipo emocional. Es decir, la información sobre riesgo no debe presentarse de forma excesivamente amenazante o cruenta, debe basarse en información veraz y relevante y debe darse importancia especial a la forma correcta de evitarlo. Las respuestas defensivas de tipo emocional surgen ante estímulos de alta intensidad o potencialmente dañinos. Por tanto, los mensajes muy agresivos podrían generar respuestas de tipo defensivo que dificultan el procesamiento de la información y la aceptación de la alternativa recomendada.

\section{¿Cómo debe ser la información que contiene?}

- La información amenazante debe ser verídica y contrastada. Además de por cuestiones éticas, por razones prácticas. Si está tergiversada, exagerada o sesgada en algún sentido terminará por detectarse. La población percibe rápidamente cuando una información parece estar manipulada en un sentido o en otro para provocar una respuesta determinada y responde en consecuencia ${ }^{30}$.

-Además, la información en que se basa la amenaza debe ser ética, relevante y verosímil para la persona. En muchas ocasiones, el fallo de los mensajes amenazadores en producir un efecto positivo no está en la utilización de la amenaza en sí, sino en que ésta se acompaña de argumentaciones irrelevantes para el sujeto, simplistas, exageradas, incongruentes, moralistas, contrarias a sus creencias o su propia experiencia, o que no aportan alternativas viables para evitar el riesgo. "La droga mata" o "El alcohol es malo para la salud", serían ejemplos de este tipo de mensajes simples o incongruentes con su experiencia, que tienen muchas probabilidades de provocar el rechazo de la audiencia.

En la mayoría de estos casos, la presentación amenazante cumpliría su primer cometido de captar la atención del sujeto, sin embargo, el análisis posterior de la información llevaría al sujeto a rechazar o minimizar el riesgo porque "no es cierto," "no me afecta", "es un intento de manipulación" o "no me ofrece alternativa".

-Los mensajes no deben limitarse a presentar información sobre riesgos sino que deben reconocer los beneficios percibidos en el consumo y tratar de contrarrestarlos. En el caso del alcohol estos beneficios tienen una gran importancia ${ }^{31} \mathrm{y}$ resulta necesario hacer contrapeso con los riesgos, ofreciendo argumentaciones sólidas y, en lo posible, relacionadas con la experiencia o conocimientos de la audiencia.

- La amenaza con un riesgo para la salud nunca debería ir en solitario en una comunicación. Debe mostrarse siempre acompañada de una respuesta eficaz para eliminarla y fácilmente ejecutable por el sujeto. Esta respuesta puede aparecer de forma más o menos explícita, consistir en un simple símbolo o ser una explicación detallada, paso por paso, de donde acudir y lo que debe hacerse ante determinada situación, pero siempre debe estar presente.

-Los mensajes deben ser cambiantes para seguir produciendo la misma respuesta atencional en los sujetos. No es conveniente utilizar siempre los mismos argumentos. Repetir una comunicación con un mismo formato y argumentación puede provocar la habituación de la audiencia, la extinción de la respuesta atencional y el descenso de la frecuencia de aceptación de las recomendaciones ${ }^{28,32}$.

- Las comunicaciones deben tener en cuenta las características de la población a la que van dirigidas. Especialmente resulta necesario atender a los siguientes factores:

- Si existe o no percepción de riesgo previo en el individuo o la población diana: Si la percepción de riesgo es baja, como sucede en los jóvenes respecto al alcohol 3,33, puede deberse a dos motivos: o bien el riesgo no ha sido presentado nunca, o los mensajes han utilizado argumento repetitivos, irrelevantes, incongruentes con otras informaciones o su propia experiencia, o faltos de alternativas. En este caso, el segundo motivo es, probablemente, el responsable. Los jóvenes reciben, como se ha dicho, gran cantidad de mensajes sobre la ausencia de riesgos o, incluso, la bondad del consumo en su medio y en su propia familia. Sin embargo, la información sobre riesgos ha sido, en muchos casos, repetitiva en forma y argumentos o irrelevante e inapropiada para esta población.

- La intensidad de la amenaza percibida por la población: Si ya se percibe un riesgo elevado, no sólo no es conveniente, sino que resulta contraproducente insistir en este aspecto. La intensidad de la amenaza debe mantenerse en un punto medio: suficiente para motivar la atención hacia las recomendaciones, pero no tan elevada como para provocar respuestas no adaptativas. Si esto se produce, es más recomendable centrarse en la eficacia de las recomendaciones propuestas y en la capacidad individual para llevarlas a cabo.

- Los conocimientos y actitudes previas: Los estímulos significativos son únicos para cada indivi- 
duo y reflejan su historia de aprendizaje particular. Por tanto, una misma comunicación podría provocar respuestas atencionales en un grupo de individuos y respuestas defensivas en otro. La dirección de estas respuestas depende de diversos factores como la edad, el sexo, la experiencia de haber estado expuestos a información similar, o variables de personalidad. En el caso que nos ocupa, sabemos cuáles son los motivos que aducen los jóvenes para beber, tenemos información acerca de sus percepciones sobre el alcohol y hemos analizado sus conductas de consumo y relacionadas ${ }^{33,34,35}$. Esto debería servirnos para ser capaces de generar mensajes veraces, relevantes y sensibles con la realidad de los jóvenes.

En resumen, estas recomendaciones parten de la necesidad de hacer frente a las dos características que presentan las comunicaciones sobre riesgos para la salud: introducir información sobre riesgo es prácticamente inevitable y su efecto presenta una ambivalencia potencial. Sin ser la solución definitiva al problema, su aplicación debería permitir un mejor acercamiento a la necesidad de transmitir información, con el objetivo de promover la adopción libre y responsable de las alternativas más saludables.

En la actualidad creemos disponer, por tanto, de conocimientos suficientes sobre la situación; hemos analizado y desmenuzado la conducta, las creencias y las actitudes de los jóvenes; sabemos dónde van, cuándo y con quién hacen qué y por qué lo hacen; y tenemos una orientación sobre cómo decirles las cosas. Tal vez lo que nos falta sea tener una idea más clara de qué es lo que queremos decirles.

\section{REFERENCIAS}

(1) Jones S, Donovan RJ. Messages in alcohol advertising targeted to youth. Australian Journal of Public Health, 2001; 25:126-131.

(2) Sánchez R. Publicidad de bebidas alcohólicas. Algunas razones para establecer unos límites. Adicciones, 2002; 14 (Supl. 1): 133-140.

(3) Calafat A, Juan M, Becoña E, Fernández C, Gil E, Palmer A, Sureda P, Torres MA. Salir de marcha y consumo de drogas. Plan Nacional sobre Drogas. Ministerio del Interior, 2001.

(4) Plan Nacional sobre Drogas. Estrategia Nacional sobre Drogas 2000-2008. 2002 PNSD, Madrid.

(5) Heather N. Intervenciones breves: Una oportunidad para reducir el consumo excesivo de alcohol entre los jóvenes. Adicciones, 2001; 13, 4.

(6) Rodríguez-Martos A. Efectividad de las técnicas de consejo breve. Adicciones, 2002; 14 (Supl. 1): 337-352.
(7) Marlatt GA, Baer, JS, Kivlahan DR, Dimeff LA, Larimer ME, Quigley LA, Somers JM, Williams E. Screening and brief intervention for high-risk collage student drinjers: results from a 2-year follow-ul assessment. Journal of Consulting and Clinical Psychology, 1998; 66 (4): 604-615.

(8) Baer JS, Kivlahan DR, Blume AW, McKnight P, Marlatt A. Brief intervention for heavy-drinking college students: 4-year follow-up ans natural history. American Journal of Public Health, 2001; 91 (8):1310-1316.

(9) Calafat A. Estrategias preventivas del abuso del alcohol. Adicciones, 2002; 14 (Supl. 1): 317-335.

(10) Ordoñana JR, Gómez-Amor J, Galvañ F. El uso del miedo en los mensajes de salud. Gaceta Sanitaria, 2000; 14 (Supl. 3):45-59.

(11) Ajzen I. Persuasive Communication Theory in Social Psychology. A Historical Perspective. En Manfredo MJ (Ed.) Influencing Human Behavior: Theory and Applications in Recreation and Tourism. Champaign, IL. Sagamore Pub, 1992.

(12) Donovan RJ, Henley N. A conceptual framework for fear arousal and threat appeals in health promotion communications. Health Promotion Journal of Australia, 2000; 10 (2): 84-88.

(13) Schneider TR, Salovey P, Pallonen U, Mundorf N, Smith NF, Steward WT. Visual and auditory message framing effects on tobacco smoking. Journal of Applied Social Psychology, 2001; 31 (4): 667-682.

(14) Witte K. Putting the fear back into fear appeals: the extended parallel process model Communication Monographs, 1992a; 59, 329-349.

(15) Witte K, Allen M. A meta-analysis of fear appeals: Implications for public health campaigns. Health Education \& Behavior, 2000; 27 (5): 591-615.

(16) Hale JL, Dillard JP. Fear appeals in health promotion campaigns: Too much, too little, or just right?. En Maibach, E; Parrot, RL (Eds.) Designing Health Messages. Approaches from Communication Theory and Public Health Practice. 65-80 Londres: Sage Publications, 1995.

(17) Boster FJ, Mongeau P. Fear-arousing persuasive messages. En Bostrom, RN; Westley, BH (Eds) Communication Yearbook, 1994; 8, 330-375.

(18) Moscato S, Black DR, Blue CL, Mattson M, Galer-Unti $\mathrm{R}$, Coster D. Evaluating a fear appeal message to reduce alcohol use among "greeks". American Journal of Health Behavior, 2001; 25 (5): 481-491.

(19) Van Weel F, Knobbout J. Adolescents and fear appeals. International Journal of Adolescence and Youth, 1998; 7: 121-135.

(20) Bennet P, Calman K. Risk Communication and Public Health. Londres: Oxford University Press, 1999.

(21) Edwards A, Elwyn G, Gwyn R. General prac tice registrar responses to the use of different risk communication tools in simulated consultations: a focus group study. British Medical Journal, 1999; 319: 749-752. 
(22) Witte K. Message and conceptual confounds in fear appeals: the role of threat, fear, and efficacy The Southern Communication Journal, 1992b; 58, 147-155.

(23) LePoire BA, Burgoon JK. Usefulness of differentiating arousal responses within communication theories: orienting response or defensive arousal within nonverbal theories of expectancy violation. Communication Monographs, 1996; 63: 208-230.

(24) Ordoñana JR. Uso del miedo en los mensajes de salud. Relación entre el nivel de activación psicofisiológica y cambios conductuales producidos por mensajes de salud sobre vacunaciones. Tesis Doctoral no publicada. Universidad de Murcia, 1997.

(25) Ordoñana JR, González F, Carrillo E, Pérez-Riquelme F. Respuesta psicofisiológica ante mensajes informativos: un proyecto de aplicación práctica a la promoción de salud (Estudio piloto). Comunicación presentada en la II International Conference on Emotion \& the Brain, Palma de Mallorca, 2001.

(26) González F, Ordoñana JR, Carrillo E, Gómez J. Effects of different kind of health messages: psychophysiological responses and health behaviors. Journal of Psychophysiology (En prensa).

(27) Gómez J, Ordoñana JR, Carrillo E, González F, García C. Los jóvenes y la prevención del consumo de alcohol, comparación de los efectos de distintos mensajes de salud. Comunicación presentada en el Primer Con- greso del Instituto Nacional de Investigación y Formación sobre Drogas. Delegación del Gobierno para el Plan Nacional sobre Drogas, Madrid: 2002.

(28) U.S. Department of Health and Human Services. Reducing Tobacco Use. A Report of the Surgeon General. Centers for Disease Control and Prevention. Atlanta, 2000

(29) Rise J, Wilhelmsen BU. Prediction of adolescents' intention not to drink alcohol: theory of planned behaviour. American Journal of Health Behavior, 1998; 22 (3): 206-217.

(30) Friestad M, Wright P. The persuasion knowledge model - How people cope with persuasion attempts. Journal of Consumer Research, 1994; 21 (1) 1-31.

(31) Goldberg JH, Halpern-Felsher BL; Millstein SG. Beyond invulnerability: The importance of benefits in adolescents' decision to drink alcohol. Health Psychology, 2002; 21 (5), 477-484.

(32) Donohew L, Palmgreen P, Lorch EP. Attention, need for sensation, and health communication campaigns. American Behavioral Scientist, 1994; 38 (2): 310-322.

(33) Sánchez L. Consumo de alcohol en la población juvenil. Adicciones, 2002; 14 (Supl. 1): 123-132.

(34) Sánchez L. Consumo alcohólico en la población española. Adicciones, 2002; 14 (Supl. 1): 79-97.

(35) Pascual F. Percepción del alcohol entre los jóvenes. Adicciones, 2002; 14 (Supl. 1): 123-131. 
